\title{
Hybrid Parameter Control Approach Applied to a Diversity-based Multi-objective Memetic Algorithm for Frequency Assignment Problems
}

\author{
Eduardo Segredo*, Ben Paechter*, Emma Hart*, Carlos Ignacio González-Vila ${ }^{\dagger}$ \\ ${ }^{*}$ School of Computing \\ Edinburgh Napier University \\ Edinburgh, Scotland, UK \\ †Instituto Tecnológico y de Energías Renovables (ITER) \\ Santa Cruz de Tenerife, Spain \\ Email: e.segredo@napier.ac.uk, b.paechter@napier.ac.uk, e.hart@napier.ac.uk, cigonzalez@iter.es
}

\begin{abstract}
In order to address the difficult issue of parameter setting within a diversity-based Multi-objective Evolutionary Algorithm (MOEA), we recently proposed a hybrid control scheme based on both Fuzzy Logic Controllers (FLCs) and Hyperheuristics (HHs). The method simultaneously adapts both symbolic and numeric parameters and was shown to be effective when controlling a diversity-based MOEA applied to a range of benchmark problems. Here, we show that the hybrid control scheme generalises to other meta-heuristics by using it to adapt several parameters of a diversity-based multi-objective Memetic Algorithm (MA) applied to a Frequency Assignment Problem (FAP). Using real-world instances of the FAP, we demonstrate that our proposed parameter control method outperforms parameter tuning of the MA. The results provide new evidence that the method can be successfully applied to significantly more complex problems than the benchmarks previously tested.
\end{abstract}

\section{INTRODUCTION}

Despite the success of Evolutionary Algorithms (EAs) in solving a wide variety of problems, the need to avoid approaches converging to local optima still remains a challenging task. Although many methods have been proposed for maintaining diversity, an approach that has recently gathered popularity is to consider the use of a multi-objective algorithm when solving a single-objective problem [1]. Additional objectives measuring diversity are introduced besides the original objective of the problem at hand. Any Multi-objective Evolutionary Algorithm (MOEA) can then be applied to solve that problem.

However, as is common with most EAs, multi-objective approaches have configurable components (e.g. choice of mutation or crossover operator) and parameters that must be appropriately set. Judicious choice of both components and parameters can have significant impact of the eventual performance of the algorithm. As a consequence, considerable research effort is focused on parameter setting [2], with approaches tending to focus on tuning of parameters or control of parameters. In the former, the goal is to pre-select the best settings, which are held at these values throughout the execution of the algorithm on a given instance. In the latter approach, an online procedure alters parameters during the course of a run, based on some guiding strategy.
In [3], Fuzzy Logic Controllers (FLCs) and Hyper-heuristics (HHs) were compared as control methods for adapting two numeric parameters of a diversity-based multi-objective Memetic Algorithm (MA) applied to a Frequency Assignment Problem (FAP). The main drawback was that both parameters were optimised independently, i.e. one parameter was adapted while the other one remained fixed for the whole run, having performed two separate studies. Afterwards, in [4], a novel hybrid control scheme that utilised both FLCs and HHs was proposed, which is able to adapt several symbolic and numeric parameters simultaneously. In that work, the hybrid scheme simultaneously controlled different parameters of a diversitybased MOEA applied to benchmark problems. Here, we extend the previous work in two directions. Firstly, we show that the hybrid control approach can be generalised in that it can simultaneously adapt multiple parameters of another meta-heuristic. Specifically, we apply it to the diversity-based multi-objective MA used for solving the FAP [4]. Secondly, we demonstrate that the hybrid control scheme is not only suitable for solving benchmark problems successfully, but is also applicable to real-world applications. The main contributions of the current research work are the following:

- The first application of a hybrid parameter adaptation scheme based on FLCs and HHs to a diversity-based multi-objective MA.

- New evidence relating to the benefits of control vs. tuning, based on an extensive comparison of the hybrid method to experiments using a diversity-based MOEA with fixed parameters (considering variable configurations).

\section{BACKGROUND IN PARAMETER CONTROL IN EVOLUTIONARY ALGORITHMS}

As previously mentioned, selecting appropriate parameters remains a challenging task within EA design [5]. Parameter-setting approaches typically consider two parameter classes [6]: numeric parameters such as mutation rates or population size (also known as quantitative or behavioural parameters), and symbolic parameters that specify operator 
selection, e.g. for crossover or selection (sometimes described as qualitative, categoric or structure parameters).

The main difference between the parameter types lies in the definition of their domains. Symbolic parameters are associated with a discrete domain in which order cannot be established, and for which a distance metric cannot be easily defined. In contrast, numeric parameters are specified by an infinite domain in which it is straightforward to both define a distance metric and to establish order.

Parameter control strategies aim to select the most appropriate parameter values for a given state of the search procedure. The notion of parameter control was first introduced in very early research on EAs [7], [8], and has rapidly expanded in recent years [2]. In addition, the range of methods to which parameter control has been successfully applied has also increased, for example including MAs [9] and Differential Evolution (DE) [10]. Several taxonomies have been proposed to categorise these approaches. One of the most popular [2] distinguishes between deterministic, adaptive, and self-adaptive control. Deterministic methods modify the parameters applying deterministic rules, without using any feedback from the search process. On the contrary, adaptive schemes use some feedback from the optimisation procedure to update the parameters. Finally, in self-adaptive approaches, parameters are encoded into the chromosome and changed during the variation stage.

More recently, the majority of work relating to parameter control has focused on parameter adaption in the variation stage, and on designing combined and parameter-independent control mechanisms [2]. Here, we describe a generic approach to parameter control that combines FLCs and HHs. The method is integrated with a diversity-based multi-objective MA, and applied to a FAP. The FLC part controls the adaptation of numeric parameters (both discrete and continuous), while the $\mathrm{HH}$ part controls adaptation of symbolic parameters, as well as discrete numeric parameters.

\section{DIVERSITY-BASED MULTI-OBJECTIVE MEMETIC ALGORITHM FOR THE FREQUENCY ASSIGNMENT PROBLEM}

In a typical FAP, a set of transceivers are installed in different sectors of a given area. Each transceiver has an associate set of valid frequencies that can be assigned to it. Interference can occur between adjacent sectors depending on the frequencies assigned to their corresponding transceivers. The objective is to assign suitable frequencies to each transceiver such that the total interference in the area is minimised.

In this section, we describe the meta-heuristic used to optimise the FAP that we apply our hybrid control method to. A formal definition of this meta-heuristic can be found in [11]. This meta-heuristic was first proposed in [12] and was selected because it yielded the best solutions considering different instances of the FAP in previous work [3], [11], [12].

The scheme is a diversity-based multi-objective MA based on the well-known NSGA-II [13]. The only one difference with regard to the original NSGA-II is that after the variation stage, a local search is applied to every newly generated individual. In diversity-based multi-objective schemes, each individual is associated with a number of objectives. Typically, the first relates to the objective function of the particular problem under consideration. In the case of the FAP, this must be minimised. The remaining objectives consist of measures of the diversity introduced by an individual itself. Note that here, as is common in other work, we only consider one additional objective. We previously tested two potential candidate metrics [3], [11]: the Distance to the Closest Neighbour (DCN) and the Average Distance to all Individuals (ADI), proposed in [14] and [15], respectively. Both of them should be maximised.

The genetic operators and the local search scheme are important components for the efficiency of the algorithm. The local search is a Lamarckian approach [16], i.e. the individual reflects in its genotype the result of the movements performed by the local search. The operation of the local search is detailed in [11], but basically it optimises the assignment of the frequencies to the transceivers located in a given sector without modifying the remaining network assignments.

The genetic operators were specifically designed to address the considered variant of the FAP. As is normal, the scheme relies on the application of variation operators, i.e. crossover and mutation, with probabilities $p_{c}$ and $p_{m}$, respectively. Two different crossover operators were tested, one of them random and one that considers problem-dependent information. Only one of both options was applied in the variation stage, depending on the FAP instance. They operate as follows:

- Uniform Crossover (UX). For each gene, a random value $r \in[0,1]$ is uniformly selected. When $r<0.5$, the gene of the first parent is inherited by the offspring. Otherwise, the gene of the second parent is considered.

- Interference-based Crossover (IX). Firstly, a transceiver $t$ is selected at random. Then, genes related to transceivers that interfere with $t$ or are interfered with by $t$, including the gene representing $t$, are taken from the first parent. For the remaining genes, the second parent is considered.

After applying one of the aforementioned crossover operators, the Neighbourhood-based Mutation (NM) operator is applied. Its function is as follows. First, a transceiver $t$ is randomly selected. Afterwards, a list called interference, which consists of the transceivers that interfere with $t$, or are interfered with by $t$, is created. Every transceiver in that list is then mutated with probability $p_{m}$. The above step is repeated $R$ times, but in the next iterations the transceiver is selected at random from among those that were included in the initial interference list. This results in mutation being directed to a specific region of the network.

One of the main drawbacks of the application of this operator is that two different numeric parameters must be set. One of these parameters $\left(p_{m}\right)$ is continuous and the other one $(R)$ is discrete. These parameters were independently adapted by the use of FLCs and HHs as control methods, and as a result, two separate studies were carried out [3]. In both studies, FLCs and HHs demonstrated high performance when controlling the parameters of the NM operator in terms of the quality of frequency plans were obtained. In this work, we go beyond this in combining FLCs and HHs in a single control approach in order to adjust $p_{m}$ and $R$ simultaneously.

Two other components must be specified. Arrays of $n$ integer values $\left(p_{1}, p_{2}, \ldots, p_{n}\right)$ are used to encode individuals, 
in which $n$ is the total number of transceivers installed in the area, and $p_{i}$ the frequency assigned to the transceiver $t_{i}$. Binary Tournament [5] is used as the parent selection mechanism.

\section{HYBRID CONTROL SCHEME}

In this section, we describe our hybrid control mechanism based on HHs and FLCs, together with their components. The parameters of the $\mathrm{NM}$ operator are adapted by this hybrid control approach.

As it can be observed in Figure 1, the control scheme consists of several layers. A selection $\mathrm{HH}$ [17], which is located at the first layer, is responsible for adapting discrete numeric parameters, as well as symbolic ones. Said HH takes into consideration the past performance of different low-level configurations with the aim of selecting the most promising one at the current stage of the search process. In this work, lowlevel configurations are defined by different parameterisations of the diversity-based MA described in Section III, each one of them with a particular value assigned to parameter $R$. At the same time, the FLC present at the second layer, adjusts the mutation rate $p_{m}$. The remaining parameters of the low-level configurations are kept constant during the whole execution. When the HH selects a particular low-level configuration, it is executed until certain local stopping criterion is satisfied. Then, another low-level configuration is selected and executed by using the final population of the last-low level configuration run as its initial population. We should note at this point that the newly selected low-level configuration might potentially be the last one executed. Finally, while a global stopping criterion is not satisfied, the above steps are repeated.

As it was mentioned before, an FLC, which is located at the second layer, is used to control numeric parameters, and in the case of this work, it is responsible for adapting the mutation rate $p_{m}$. In order to carry out new decisions, it considers historical information regarding previously inferred parameter values. In this second layer, the selected low-level configuration is run until the $\mathrm{HH}$ local stopping criterion is reached. The FLC, however, has to periodically calculate new values for parameter $p_{m}$ as well, and consequently, it defines another local stopping criterion. An example clarifies: if $1.5 \times 10^{5}$ evaluations are considered as the global stopping criterion, and the $\mathrm{HH}$ local stopping criterion is fixed to $3 \times 10^{3}$ evaluations, then the HH performs 50 decisions for the whole run, by selecting different low-level configurations with different values for parameter $R$. Every selected low-level configuration is executed during $3 \times 10^{3}$ evaluations. Moreover, if the FLC local stopping criterion is fixed to to $1.5 \times 10^{2}$ evaluations, then the FLC calculates 20 different values for the mutation rate $p_{m}$ during each execution of a low-level configuration selected by the HH.

\section{A. Hyper-heuristic}

In this work, we consider a version of the selection $\mathrm{HH}$ introduced by [18] for altering parameter $R$ of the NM operator. We selected this HH since in previous work it has been applied with success [3], [19]. It selects the most appropriate lowlevel configuration by making use of scoring and selection mechanisms. The selection mechanism consists of the following steps. In first place, each low-level configuration is scored

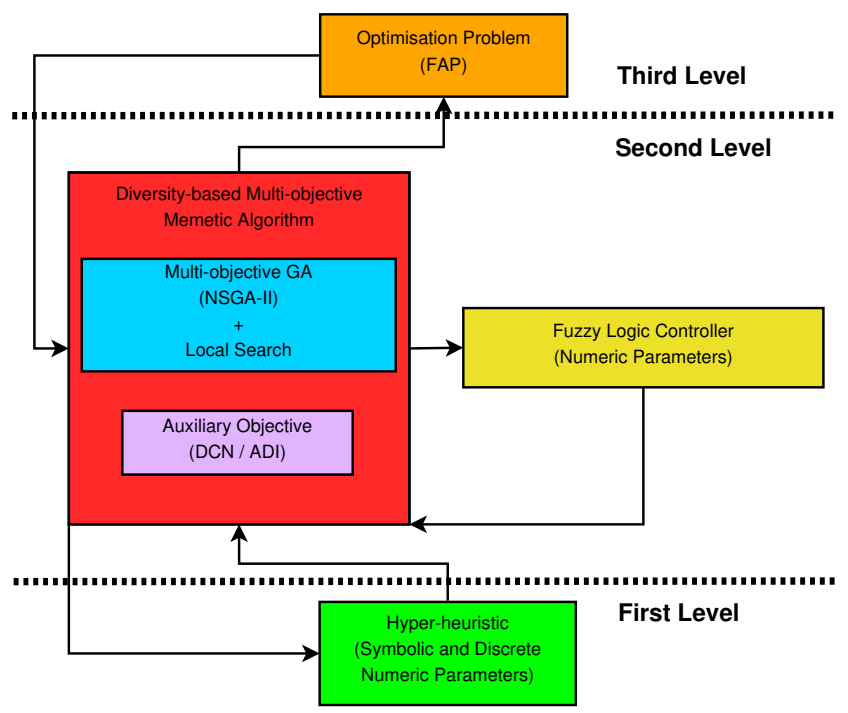

Figure 1. Different layers and components of the hybrid control approach

by the scoring mechanism. The goal of that approach is to estimate the improvement that every low-level configuration might potentially provide considering the current population. Hence, the higher the score, the more promising the low-level configuration. Scores are calculated taking into account the historical improvements in the original objective value, i.e. in the FAP cost function, provided by every low-level configuration. The difference between the best obtained individual and the best initial individual, in terms of the original objective value is defined as the improvement $\gamma$. Therefore, score $s($ conf $)$ is calculated as a weighted average of the last $k$ improvements obtained by configuration conf:

$$
s(\operatorname{con} f)=\frac{\sum_{i=1}^{\min (k, j)}(\min (k, j)+1-i) \cdot \gamma[\operatorname{con} f][j-i]}{\sum_{i=1}^{\min (k, j)} i}
$$

In (1), the improvement obtained by conf in run $j-i$ is represented by $\gamma[\operatorname{con} f][j-i]$. The value of $k$ allows the adaptation level of the HH to be defined. The adaptation level is the quantity of historical information taken into consideration by the HH to carry out its decisions. Finally, it can be observed that a greater importance is assigned to the most recent runs.

The HH makes use of a parameter called $\beta$, which defines the minimum selection probability of every low-level configuration. Thus, $\beta \cdot n_{h}$ percentage of the decisions, with $n_{h}$ being the number of low-level configurations used, are randomly performed considering a uniform distribution. Therefore, every low-level configuration conf is selected with a probability calculated as shown in (2).

$$
\operatorname{prob}(\operatorname{conf})=\beta+\left(1-\beta \cdot n_{h}\right) \cdot\left[\frac{s(\operatorname{conf})}{\sum_{i=1}^{n_{h}} s(i)}\right]
$$


In this work, we consider two different approaches based on the above $\mathrm{HH}$, an elitist one (HH-ELI) and a probabilistic one (HH-PROB). The former always selects the configuration with the maximum score $s(\operatorname{con} f)$, in addition to the minimum number of decisions randomly performed for every configuration. In the case of the latter, the selection probability is calculated as shown in (2).

\section{B. Fuzzy logic controller}

The FLC proposed to control parameter $p_{m}$ of the NM operator is described in this section. It makes use of several fuzzy rule bases, and at the current stage of the search, a different rule base, which is responsible for guiding the adaptation of the mutation rate $p_{m}$, is enabled by considering historical data. Algorithm 1 shows the pseudocode of our FLC.

Steps 1-4, i.e. initialisation and learning stages, are executed if and only if it is the first time that a particular lowlevel configuration is selected by the $\mathrm{HH}$. At the same time, both the state of the FLC and the state of the current low-level configuration are stored once the $\mathrm{HH}$ local stopping criterion is met. Hence, the last state of a low-level configuration that had been previously run is restored before the start of its new execution. We should note that Mamdani's fuzzy inference is applied during the fuzzy inference process (lines 8-10). Moreover, the fuzzy logic operator AND and the implication method apply the minimum T-norm, the aggregation method applies the maximum S-norm, and the centroid algorithm is used as the defuzzification method. Since all of these components are frequently used when applying Mamdani-type FLCs, we selected them for our implementation.

We define the following input variables (line 6):

- IMP. The improvement, in terms of the original objective value of the best solution, provided by the diversity-based MA (line 12) considering the latest numEvals function evaluations. It is enclosed in the range $[0,1]$. Note that numEvals represents the local stopping criterion established by the FLC.

- VAR. It measures the population diversity. The larger its value, the higher the diversification of individuals. This variable is calculated as shown in (3). Terms $x_{j}[i]$ and $x_{k}[i]$ denote the decision variable $i$ of individuals $j$ and $k . D$ and $N$ represent the number of decision variables and the population size, respectively. VAR* is enclosed in range $[0,1]$ to normalise VAR.

$$
\mathrm{VAR}^{*}=\sum_{i=0}^{D-1}\left[\sum_{j=0}^{N-1}\left[x_{j}[i]-\frac{1}{N} \cdot\left(\sum_{k=0}^{N-1} x_{k}[i]\right)\right]^{2}\right]
$$

- PM-IN. Enclosed in the range $[0,1]$, it represents the current value of the mutation rate $p_{m}$.

- $\quad$ BEST-PM-IN. It is defined as that value of the mutation rate $p_{m}$ that has been able to provide the maximum improvement, in terms of the original objective value of the best individual, taking into account the latest $k$ decisions carried out by the FLC. It is also enclosed in the range $[0,1]$.

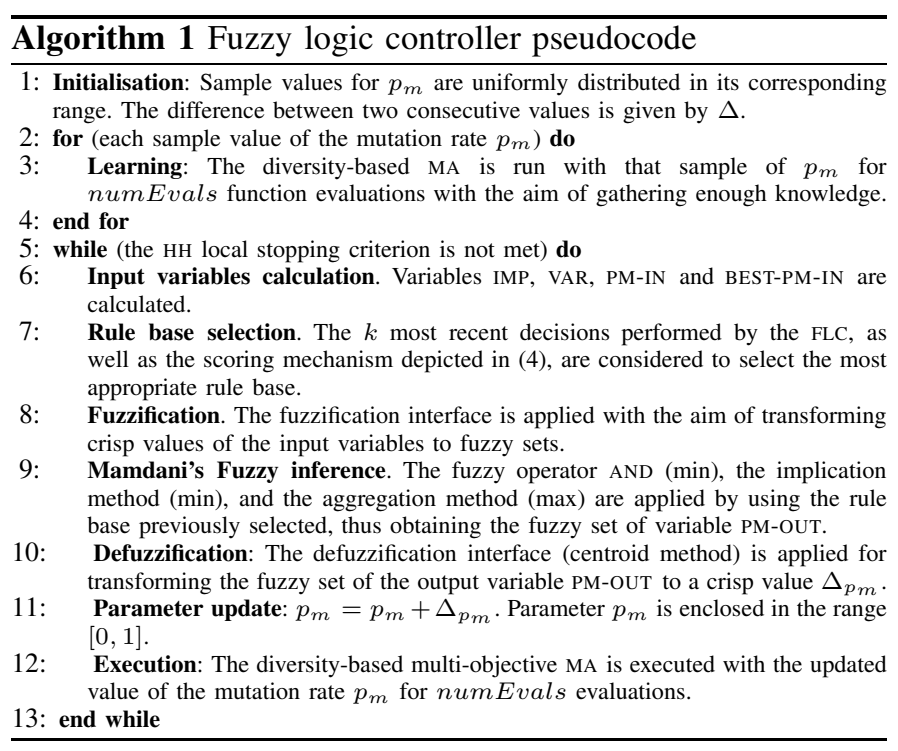

In this work, we consider two variants of the above FLC. The first one, which uses the input variables IMP, VAR and PMIN, is named FUZZY-A, while the second one, which makes use of the input variables IMP, PM-IN and BEST-PM-IN, is called FUZZY-B. We have defined only one output variable for both variants. It is used to calculate the increment or decrement to be applied to the mutation rate $p_{m}$ to update its current value, and is named PM-OUT. Figure 2 shows the membership functions of the aforementioned variables. We selected triangular-shaped membership functions because of their computational simplicity and efficiency.

Both versions of the FLC make use of several fuzzy rule bases. Depending on the behaviour of the FLC in previous runs, a different set of fuzzy rules will be applicable. For example, consider that the best performance has been obtained by low values of the mutation rate $p_{m}$. The usage of those low values should be then promoted by the selected rule base. Different IF-THEN rules define a rule base. One of the rule bases belonging to the approach FUZZY-A is described in Table I. The remaining rule bases, including those belonging to FUZZY-B, are not shown due to space constraints. The reader is referred to [3] to find the complete definition of the rule bases for both FLCs. We should note that three input variables and one output variable are considered for each fuzzy rule, and the antecedents of those rules only take into account the fuzzy logic operator AND. A particular fuzzy rule has no dependency on a particular variable when a '-' is shown.

As in the case of the $\mathrm{HH}$, a scoring mechanism is used to select the most appropriate rule base at the current stage of the search. Two different types of information are considered by that scoring function: information about the membership degree of the current value of $p_{m}$ to each linguistic term of the variable PM-IN, and data about the improvement achieved in the original objective value. The FLC takes into account historical knowledge regarding the most recent $k$ decisions carried out. Parameter $k$ allows the amount of historical data considered to be tuned. Each linguistic term $i \in[0$, numTerms -1$]$ is assigned a score calculated by (4), with numTerms being the number of linguistic terms of the variable PM-IN, and $d$ the quantity of inferences carried out by the FLC. The term $\gamma[d-j]$ 
Membership Functions - IMP, VAR and BEST-PM-IN

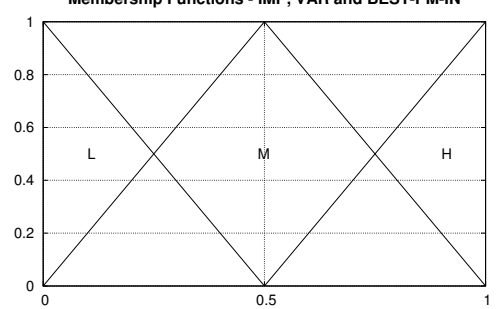

Membership Functions - PM-IN

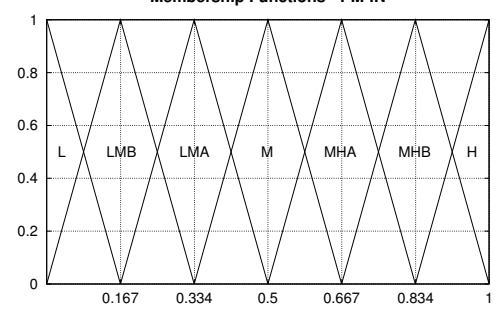

Membership Functions - PM-OUT

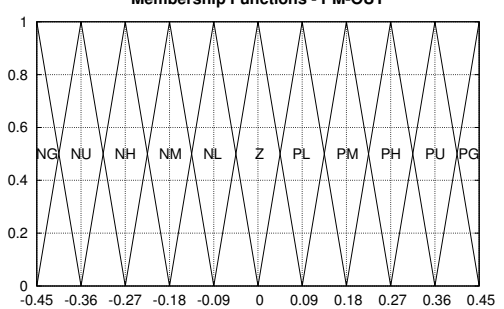

Figure 2. Definition of membership functions for the fuzzy logic controller variables

represents the improvement achieved by the diversity-based multi-objective MA in execution $d-j$ (line 12 of Algorithm 1). Moreover, $\delta[i][d-j]$ denotes the degree of membership of $p_{m}$ to the term $i$ in execution $d-j$. A higher score will be thus assigned to the linguistic term $i$ if values inferred for the mutation rate $p_{m}$ provide larger improvements in the original objective, and at the same time, if they have higher degrees of membership to that term. Finally, it can be observed that a higher importance is assigned to the last decisions performed.

$$
\operatorname{score}[i]=\frac{\sum_{j=1}^{\min (k, d)} \gamma[d-j] \cdot \delta[i][d-j] \cdot(\min (k, d)-j+1)}{\sum_{j=1}^{\min (k, d)} \delta[i][d-j] \cdot(\min (k, d)-j+1)}
$$

It is important to remark that the above scoring function will work if and only if numTerm rule bases are defined, thus meaning that variable PM-IN consists of numTerms linguistic terms. As it is shown in Figure 2, variable PMIN consists of seven terms, and therefore, we implemented seven different rule bases. Several numbers of rule bases were tested. The higher that number, the smoother the variations of $p_{m}$ inferred by the FLC, and thus the steadier the FLC. Nevertheless, when considering a higher quantity of rule bases, as well as with a lower amount of them, the performance of the whole optimisation scheme decreased. Three terms were defined for the rest of input variables with the aim of keeping rule bases as simple as possible.

The linguistic term $i$ that maximises the scoring function is selected, and consequently, rule base $i$ is enabled. Hence, parameter values of $p_{m}$ with high degrees of membership to the term $i$ should achieve better solutions than those provided by other values, and rule base $i$ is responsible for adapting the mutation rate $p_{m}$ so that it approaches the values represented by linguistic term $i$. For example, consider that the current value of $p_{m}$ is 0.9 and that we are using the approach FUZZYA. Once scores are calculated, assume that the selected rule base is that associated to the term LOW (L) of the variable PM-IN. Bearing the above in mind, low values of $p_{m}$ have historically achieved significant improvements in terms of the original objective. Table I shows the selected rule base for this particular case. If a fuzzy set for the variable IMP, which has a large degree of membership to the term LOW (L), since PMIN (with value 0.9) is represented by a fuzzy set with a large degree of membership to the term HIGH $(\mathrm{H})$, then the output fuzzy set, i.e the one corresponding to PM-OUT, will have a large degree of membership to the linguistic term NEG-GIANT
Table I. EXAMPLE OF A RULE BASE BELONGING TO FUZZY-A

\begin{tabular}{c|ccc|c} 
Rules & \multicolumn{3}{|c|}{ Input variables } & Output variables \\
\hline ID & PM-IN & IMP & VAR & PM-OUT \\
\hline 1 & L & L & L & PL \\
\hline 2 & L & L & M & PL \\
\hline 3 & L & L & H & NL \\
\hline 4 & L & M & - & Z \\
\hline 5 & L & H & - & Z \\
\hline 6 & LMB & L & - & NM \\
\hline 7 & LMB & M & - & NL \\
\hline 8 & LMB & H & - & Z \\
\hline 9 & LMA & L & - & NH \\
\hline 10 & LMA & M & - & NL \\
\hline 11 & LMA & H & - & Z \\
\hline 12 & M & L & - & NU \\
\hline 13 & M & M & - & NL \\
\hline 14 & M & H & - & Z \\
\hline 15 & MHA & L & - & NG \\
\hline 16 & MHA & M & - & NL \\
\hline 17 & MHA & H & - & Z \\
\hline 18 & MHB & L & - & NG \\
\hline 19 & MHB & M & - & NL \\
\hline 20 & MHB & H & - & Z \\
\hline 21 & H & L & - & NG \\
\hline 22 & H & M & - & NL \\
\hline 23 & H & H & - & Z
\end{tabular}

(NG). The value of the mutation rate $p_{m}$ will be thus decreased in a significant way so that it will approach lower values.

\section{EXPERIMENTAL EVALUATION}

This section is devoted to describe experiments conducted with the original MA (Experiment 1), and with the MA combined with the proposed control method (Experiment 2).

a) Experimental Method: The aforementioned approaches were implemented using the Meta-heuristic-based Extensible Tool for Cooperative Optimisation (METCO) [20]. Experiments were run on Teide High Performance Computing facilities composed of 1100 Fujitsu $\AA$ computer servers, with a total of 17800 computing cores and 36 TB of memory. Each computing node has two Intel $\mathrm{R}$ Xeon ${ }^{\mathrm{TM}}$ E5-2670 processors with 8 cores, and 32 GB RAM DDR-3. The FLCs were implemented using the library fuzzylite 5.0 [21], while GCC 4.8.2 was the compiler version considered. Since we applied stochastic algorithms, every execution was run 32 times. Statistical comparisons were carried out by performing a statistical procedure, which is explained below. First, a Shapiro-Wilk test was performed to check whether the values of the results followed a normal (Gaussian) distribution or not. If so, the Levene test checked for the homogeneity of the variances. If the samples had equal variance, an ANOVA test was done. Otherwise, a Welch test was performed. For non-Gaussian distributions, the non-parametric Kruskal-Wallis 
Table II. CONFIGURATION OF THE DIVERSITY-BASED MULTI-OBJECTIVE MEMETIC ALGORITHM

\begin{tabular}{llll}
\hline Parameter & Value & Parameter & Value \\
\hline Stopping criterion & $1.5 \times 10^{5}$ evals. & Crossover rate $\left(p_{c}\right)$ & 1 \\
Population size $(N)$ & 10 individuals & Mutation rates $\left(p_{m}\right)$ & $0,0.2,0.4,0.6,0.8,1$ \\
Crossover operators & UX (Seattle), IX (Denver) & NM operator steps $(R)$ & $1,2,3, \ldots, 14,15$ \\
Auxiliary objective & DCN (Seattle), ADI (Denver) & & \\
\hline
\end{tabular}

Table III. CONFIGURATION OF THE HYPER-HEURISTICS HH-ELI AND HH-PROB

\begin{tabular}{llll}
\hline Parameter & Value & Parameter & Value \\
\hline Local stopping criterion & $1.5 \times 10^{3}, 3 \times 10^{3}$ evals. & Minimum selection rate $(\beta)$ & 0.1 \\
Number of low-level configs. $\left(n_{h}\right)$ & 15 configs. & Historical knowledge $(k)$ & 2 \\
\hline
\end{tabular}

Table IV. CONFIGURATION OF THE FUZZY LOGIC CONTROLLERS FUZZY-A AND FUZZY-B

\begin{tabular}{llll}
\hline Parameter & Value & Parameter & Value \\
\hline Local stopping criterion $($ numEvals $)$ & $1.5 \times 10^{2}$ evals. & Difference among samples $(\Delta)$ & 0.1 \\
Number of linguistic terms (numTerms) & 7 & Historical knowledge $(k)$ & 2 \\
Range of the parameter $p_{m}$ & {$[0,1]$} & & \\
\hline
\end{tabular}

test was used. All statistical tests were applied considering a significance level equal to $5 \%$.

b) FAP instances: The studies were conducted considering two different instances representing two real cities in the USA: Seattle and Denver. The Seattle instance had $n=$ 970 transceivers and 15 different frequencies to be assigned. The Denver instance was larger, consisting of $n=2612$ transceivers and 18 frequencies. The remaining parameters belonging to both instances were set as in [3].

c) Parameters for Experiment 1: Parameter values for the diversity-based multi-objective MA are shown in Table II. A total number of 90 configurations of the diversity-based multi-objective MA with fixed parameters were executed for each instance. The values depicted in Table II for parameters $p_{m}$ and $R$ were combined in order to obtain those configurations. A different crossover operator, as well as a particular auxiliary objective, were considered depending on the instance. Moreover, it is important to remark that 10 individuals were taken into account as the population size. We selected those parameter values because we obtained the best solutions when applying them together with the diversitybased multi-objective MA to the FAP [3], [12]. Finally, the different stopping criteria were set in terms of the total amount of evaluations carried out, since one of the most expensive operations from the computational view is the calculation of the evaluation function. In order to identify a particular configuration of the diversity-based multi-objective MA, the values for $p_{m}$ and $R$ reflect the name of the scheme. For example, Fixed_0.6_13 is a fixed configuration of the diversitybased memetic approach executed with values 0.6 and 13 for the parameters $p_{m}$ and $R$, respectively.

d) Parameters for Experiment 2: This experiment was focused on executing the hybrid control scheme to simultaneously adjust the values of the parameters $p_{m}$ and $R$. The configurations of the HHs and FLCs are described in Tables III and IV, respectively. Table II shows the values used for the remaining parameters of the MA. We should note that $n_{h}=15$ low-level configurations, each one of them with a different value for the parameter $R$ (Table II), defined the candidate set of the HHs. Therefore, the only one difference among the low-level configurations lied in the particular value given to that parameter. Finally, it is worth pointing out that two local stopping criteria were taking into account for the HHs, thus giving two different configurations of the schemes HH-ELI and HH-PROB. Bearing the above in mind, and considering the FLCS FUZZY-A and FUZZY-B, 8 different configurations of the hybrid control scheme were executed for each FAP instance. In order to identify a particular configuration of the hybrid scheme, the value of the HH local stopping criterion reflects the name of the approach. For instance, FUZZY-A_HH-ELI_3000 is a configuration of the hybrid scheme that combines the schemes FUZZY-A and HH-ELI with a local stopping criterion for the $\mathrm{HH}$ equal to $3 \times 10^{3}$ evaluations.

The main goal of both experiments was twofold. First, to analyse the performance and robustness of the hybrid control mechanism. Second, to show if some advantages arise when controlling $p_{m}$ and $R$ with respect to tuning them.

Tables V and VI show different statistics, including dispersion measures (Standard Deviation-SD, Coefficient of Variation-CV), for the different configurations of the hybrid control approach, as well as for several configurations of the diversity-based multi-objective MA executed with fixed parameters, considering the Seattle and Denver instances, respectively. In the case of the diversity-based multi-objective MA executed with fixed parameters, the 90 configurations were sorted in ascending order considering the mean of the original objective value achieved by each one. Tables show information for the fixed configurations located at positions 1, 20, 40, 60, 80 , and 90 , the latter being the configuration that achieved the highest mean of the original objective value. Additionally, the last three columns show the number of fixed configurations of the diversity-based multi-objective MA that were statistically outperformed $(\uparrow)$ by, that did not present statistically significant differences $(\leftrightarrow)$ with, and that were able to outperform $(\downarrow)$ the approach located at the corresponding row, as determined by the statistical procedure described at the beginning of the 


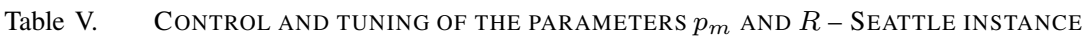

\begin{tabular}{|c|c|c|c|c|c|c|c|c|c|c|c|}
\hline Approach & Min. & First Qu. & Median & Mean & Third Qu. & Max. & SD & $\mathrm{CV}$ & $\uparrow$ & $\leftrightarrow$ & $\downarrow$ \\
\hline Fuzzy-A_HH-Eli_1500 & 526.9 & 613.5 & 643.4 & 671.4 & 736.3 & 825.5 & 81.6 & 12.2 & 45 & 45 & 0 \\
\hline Fuzzy-B_HH-Eli_1500 & 558.5 & 619.0 & 662.5 & 670.3 & 723.0 & 866.7 & 69.8 & 10.4 & 46 & 44 & 0 \\
\hline Fuzzy-A_HH-Eli_3000 & 499.3 & 637.0 & 676.0 & 679.3 & 717.4 & 876.5 & 75.8 & 11.2 & 42 & 48 & 0 \\
\hline Fuzzy-B_HH-Eli_3000 & 516.6 & 611.5 & 675.7 & 672.9 & 722.7 & 859.4 & 87.6 & 13.0 & 43 & 47 & 0 \\
\hline Fuzzy-A_HH-Prob_1500 & 520.2 & 619.5 & 679.5 & 667.6 & 718.8 & 827.0 & 78.1 & 11.7 & 48 & 42 & 0 \\
\hline Fuzzy-B_HH-Prob_1500 & 545.7 & 621.4 & 680.7 & 673.3 & 727.9 & 855.7 & 72.9 & 10.8 & 44 & 46 & 0 \\
\hline Fuzzy-A_HH-Prob_3000 & 502.5 & 582.4 & 640.2 & 649.9 & 688.5 & 888.3 & 90.8 & 13.9 & 69 & 21 & 0 \\
\hline Fuzzy-B_HH-Prob_3000 & 505.0 & 593.1 & 649.6 & 650.7 & 680.5 & 835.5 & 77.5 & 11.9 & 71 & 19 & 0 \\
\hline Fixed_0.2_12 & 512.9 & 594.8 & 646.0 & 643.9 & 673.6 & 848.6 & 75.0 & 11.6 & 79 & 11 & 0 \\
\hline Fixed_0.2_8 & 538.4 & 644.3 & 682.5 & 688.6 & 727.0 & 843.5 & 75.9 & 11.0 & 38 & 51 & 1 \\
\hline Fixed_0.4_9 & 582.3 & 661.2 & 683.4 & 703.1 & 739.0 & 834.2 & 63.3 & 9.0 & 30 & 53 & 7 \\
\hline Fixed_0.6_14 & 638.0 & 677.4 & 734.5 & 741.9 & 788.7 & 868.5 & 66.5 & 8.9 & 14 & 36 & 40 \\
\hline Fixed_0.8_12 & 645.0 & 748.7 & 800.1 & 804.2 & 857.7 & 989.6 & 80.2 & 9.9 & 7 & 13 & 70 \\
\hline Fixed_1_15 & 903.8 & 1043.0 & 1114.0 & 1102.0 & 1167.0 & 1262.0 & 91.1 & 8.3 & 0 & 1 & 89 \\
\hline
\end{tabular}

Table VI. CONTRol AND tUning of The PARAMETERS $p_{m}$ AND $R-$ DenVER INSTANCE

\begin{tabular}{|c|c|c|c|c|c|c|c|c|c|c|c|}
\hline Approach & Min. & First Qu. & Median & Mean & Third Qu. & Max. & SD & $\mathrm{CV}$ & $\uparrow$ & $\leftrightarrow$ & $\downarrow$ \\
\hline Fuzzy-A_HH-Eli_1500 & 84058.5 & 84719.5 & 85211.4 & 85265.2 & 85628.7 & 86964.9 & 751.4 & 0.9 & 63 & 27 & 0 \\
\hline Fuzzy-B_HH-Eli_1500 & 83784.2 & 84768.3 & 85208.4 & 85202.9 & 85538.5 & 87190.0 & 747.0 & 0.9 & 70 & 20 & 0 \\
\hline Fuzzy-A_HH-Eli_3000 & 84196.2 & 84946.4 & 85211.5 & 85369.6 & 85849.8 & 86917.3 & 703.2 & 0.8 & 51 & 39 & 0 \\
\hline Fuzzy-B_HH-Eli_3000 & 84309.8 & 84704.2 & 84974.0 & 85372.6 & 85923.0 & 87882.3 & 936.4 & 1.1 & 62 & 28 & 0 \\
\hline Fuzzy-A_HH-Prob_1500 & 84274.7 & 84799.0 & 85031.0 & 85232.3 & 85416.0 & 87771.4 & 782.9 & 0.9 & 79 & 11 & 0 \\
\hline Fuzzy-B_HH-Prob_1500 & 84268.5 & 84947.0 & 85225.0 & 85362.8 & 85658.1 & 87070.6 & 679.9 & 0.8 & 55 & 35 & 0 \\
\hline Fixed_0.4_15 & 84187.2 & 84765.6 & 85075.2 & 85230.5 & 85641.8 & 87489.8 & 742.4 & 0.9 & 73 & 17 & 0 \\
\hline Fixed_0.6_4 & 84531.8 & 85022.7 & 85540.1 & 85569.5 & 86030.8 & 87626.7 & 749.7 & 0.9 & 34 & 55 & 1 \\
\hline Fixed_0.4_8 & 84240.4 & 85118.6 & 85966.5 & 85764.8 & 86442.2 & 87507.3 & 884.6 & 1.0 & 24 & 62 & 4 \\
\hline Fixed_1_1 & 84964.3 & 85470.2 & 85979.5 & 86084.4 & 86505.2 & 88195.4 & 830.8 & 0.9 & 20 & 36 & 34 \\
\hline Fixed_0_6 & 85713.7 & 86866.2 & 87215.9 & 87224.5 & 87578.6 & 89008.5 & 699.1 & 0.8 & 2 & 15 & 73 \\
\hline
\end{tabular}

current section. Approach $A$ statistically outperforms scheme $B$ if there exist statistically significant differences between them, and if at the same time, $A$ provides a lower mean and median of the original objective value than $B$. Finally, the data in bold show, for each method, the configuration that achieved the lowest mean of the original objective value.

We make the following observations. With regard to parameter tuning, the configuration of the diversity-based multiobjective MA that provided the lowest mean of the original objective value applied the values 0.2 and 12 for the parameters $p_{m}$ and $R$, while in the case of Denver, the values were equal to 0.4 and 15 . In addition, these configurations exhibited statistically significant differences when compared to others. For example, taking into consideration the Seattle instance, the best-behaved fixed configuration was able to statistically outperform other 79 fixed configurations. In the case of Denver, the best fixed configuration was statistically better than other 73 configurations of the diversity-based MA executed with fixed parameters. As a result, the above confirms that the most appropriate values for a set of parameters depend on the problem and/or instance being solved.

Considering the hybrid control approach, we note that no significant differences were observed between the 8 different configurations, on both FAP instances. This demonstrates that the hybrid control scheme is robust from the point of view of its components and parameters, since if we modify them, its performance is not going to change significantly.

The final three columns of Tables V and VI enable a comparison of the parameter control and parameter tunning methods. Taking into account the Seattle instance, two parameterisations of the hybrid control scheme (FUZZY-A_HHPROB_3000 and FUZZY-B_HH-PROB_3000) were able to statistically outperform more than 65 configurations of the diversitybased multi-objective MA executed with fixed parameters. Moreover, in those cases, the hybrid control scheme did not show statistically significant differences with the remaining fixed configurations of the diversity-based multi-objective MA. Finally, it is important to mention that no fixed configuration of the diversity-based MA was able to statistically outperform any parameterisation of the hybrid control approach. The above is even more noticeable in the case of the Denver instance. Hence, the benefits of using parameter control techniques instead of using parameter tuning are significant. With only one execution of the hybrid control scheme we are able to provide similar or even better frequency plans for the FAP than those obtained using the best-behaved configuration of the diversitybased multi-objective MA. In order to find such a best-behaved configuration, we had to execute 90 different parameterisations for each instance, which involved a total number of 44500 computational hours, approximately. Bearing this in mind, the advantages of control over tuning are even higher.

Finally, it is worth pointing out that if we consider the times invested by the best performing fixed configuration (Fixed_0.2_12) and the best-behaved parameterisation of the hybrid control scheme (FUZZY-A_HH-PROB_3000) in their 32 corresponding executions for the Seattle instance, the median of the execution times were 28294.49 and 27323.41 seconds, 
respectively. We expected that the hybrid control approach introduced some computational overhead. However, we found the contrary to be true. This is because the execution time directly depends on the values assigned to the parameters $p_{m}$ and $R$. The higher the values assigned to those parameters, the higher the time invested by the NM operator, and therefore, the higher the execution time. Since the hybrid control scheme dynamically updates the values for $p_{m}$ and $R$ during the execution, it was able to provide similar results than those obtained by the best fixed configuration for the Seattle instance, but by employing a lower amount of time. Taking into consideration the the Denver instance, the same behaviour appeared.

\section{CONCLUSIONS AND FUTURE WORK}

A control scheme which hybridises an FLC and a $\mathrm{HH}$ is applied in this work to concurrently adjust several numeric parameters of a diversity-based multi-objective MA specifically designed for dealing with a FAP. Particularly, two parameters ( $p_{m}$ and $R$ ) belonging to the variation stage of the diversitybased approach are adapted. We should note this is the first time that several parameters of a diversity-based multiobjective MA are simultaneously adapted by the use of a control scheme based on FLCs and HHs.

The extensive experimental evaluation carried out over two different instances of the FAP reveal that the hybrid control approach provides similar or even better frequency plans than those provided by a significant number of fixed configurations of the diversity-based multi-objective MA. The fact that better results are returned by the hybrid control method with respect to the configurations of the diversity-based multi-objective MA executed with fixed parameters also emphasise the benefits of parameter control. Additionally, we show that the hybrid control approach is not only suitable for dealing with benchmark problems, but also for real-world applications like the FAP. Finally, we demonstrate the generality of our control approach, which is able to successfully control different types of numeric and symbolic parameters of different meta-heuristics.

Currently, our hybrid control scheme is prepared to dynamically adjust only one continuos numeric parameter, thanks to the use of the FLC. Therefore, a promising line of research would be that the control mechanism was able to adapt different continuous numeric parameters simultaneously. Another interesting future line of work would be to analyse the computational overhead introduced by the hybrid control scheme when it is applied to different types of benchmarks and real-world applications.

\section{ACKNOWLEDGEMENT}

The authors wish to acknowledge the contribution of Teide High-Performance Computing facilities to the results of this research. TeideHPC facilities are provided by the Instituto Tecnológico y de Energías Renovables (ITER, S.A).

\section{REFERENCES}

[1] C. Segura, C. A. Coello Coello, G. Miranda, and C. León, "Using multiobjective evolutionary algorithms for single-objective optimization," $4 O R$, vol. 11, no. 3, pp. 201-228, 2013.

[2] G. Karafotias, M. Hoogendoorn, and A. Eiben, "Parameter control in evolutionary algorithms: Trends and challenges," IEEE Trans. Evol. Comput., vol. 19, no. 2, pp. 167-187, April 2015.
[3] E. Segredo, C. Segura, and C. León, "Fuzzy logic-controlled diversitybased multi-objective memetic algorithm applied to a frequency assignment problem," Engineering Applications of Artificial Intelligence, vol. 30, pp. $199-212,2014$

[4] - "Control of numeric and symbolic parameters with a hybrid scheme based on fuzzy logic and hyper-heuristics," in 2014 IEEE Congress on Evolutionary Computation (CEC), July 2014, pp. 18901897.

[5] A. E. Eiben and J. Smith, Introduction to Evolutionary Computing, ser. Natural Computing Series. Springer, 2003.

[6] S. K. Smit and A. E. Eiben, "Comparing parameter tuning methods for evolutionary algorithms," in Proceedings of the Eleventh Congress on Evolutionary Computation, ser. CEC'09. Piscataway, NJ, USA: IEEE Press, 2009, pp. 399-406.

[7] L. Davis, "Adapting operator probabilities in genetic algorithms," in Proceedings of the Third International Conference on Genetic Algorithms. San Francisco, CA, USA: Morgan Kaufmann Publishers Inc., 1989, pp. 61-69.

[8] I. Rechenberg, Evolutionsstrategie: optimierung technischer systeme nach prinzipien der biologischen evolution. Frommann-Holzboog, 1973.

[9] G. Francesca, P. Pellegrini, T. Stützle, and M. Birattari, "Off-line and on-line tuning: A study on operator selection for a memetic algorithm applied to the qap," in Evolutionary Computation in Combinatorial Optimization, ser. Lecture Notes in Computer Science, P. Merz and J.-K. Hao, Eds. Springer Berlin Heidelberg, 2011, vol. 6622, pp. 203214.

[10] A. K. Qin, V. L. Huang, and P. N. Suganthan, "Differential evolution algorithm with strategy adaptation for global numerical optimization," IEEE Trans. Evol. Comput., vol. 13, no. 2, pp. 398-417, Apr. 2009.

[11] C. Segura, E. Segredo, and C. León, "Scalability and robustness of parallel hyperheuristics applied to a multiobjectivised frequency assignment problem," Soft Computing, vol. 17, no. 6, pp. 1077-1093, 2013.

[12] E. Segredo, C. Segura, and C. Leon, "A multiobjectivised memetic algorithm for the Frequency Assignment Problem," in 2011 IEEE Congress on Evolutionary Computation (CEC), june 2011, pp. 1132 -1139 .

[13] K. Deb, A. Pratap, S. Agarwal, and T. Meyarivan, "A fast and elitist multiobjective genetic algorithm: NSGA-II," IEEE Trans. Evol. Comput., vol. 6, pp. 182-197, 2002.

[14] A. Toffolo and E. Benini, "Genetic diversity as an objective in multiobjective evolutionary algorithms," Evol. Comput., vol. 11, pp. 151-167, May 2003.

[15] L. Bui, H. Abbass, and J. Branke, "Multiobjective optimization for dynamic environments," in The 2005 IEEE Congress on Evolutionary Computation, vol. 3, September 2005, pp. 2349 - 2356 Vol. 3.

[16] L. D. Whitley, V. S. Gordon, and K. E. Mathias, "Lamarckian evolution, the baldwin effect and function optimization," in Proceedings of the International Conference on Evolutionary Computation. The Third Conference on Parallel Problem Solving from Nature: Parallel Problem Solving from Nature. London, UK: Springer-Verlag, 1994, pp. 6-15.

[17] E. K. Burke, M. Gendreau, M. Hyde, G. Kendall, G. Ochoa, E. Ozcan, and R. Qu, "Hyper-heuristics: a survey of the state of the art," J Oper Res Soc, vol. 64, no. 12, pp. 1695-1724, Dec 2013.

[18] T. Vink and D. Izzo, "Learning the best combination of solvers in a distributed global optimization environment," in Proceedings of Advances in Global Optimization: Methods and Applications (AGO), Mykonos, Greece, June 2007, pp. 13-17.

[19] C. Segura, E. Segredo, and C. León, "Analysing the robustness of multiobjectivisation approaches applied to large scale optimisation problems," in EVOLVE- A Bridge between Probability, Set Oriented Numerics and Evolutionary Computation, ser. Studies in Computational Intelligence. Springer Berlin Heidelberg, 2013, vol. 447, pp. 365-391.

[20] C. León, G. Miranda, and C. Segura, "METCO: A Parallel Plugin-Based Framework for Multi-Objective Optimization," International Journal on Artificial Intelligence Tools, vol. 18, no. 4, pp. 569-588, 2009.

[21] J. Rada-Vilela, "Fuzzylite: a fuzzy logic control library and application,' 2016. [Online]. Available: http://www.fuzzylite.com 